\title{
Electric potential variations associated with yearly lake level variations
}

\author{
Frédéric Perrier, Michaël Trique, Benoit Lorne, and Jean-Philippe Avouac \\ Laboratoire de Détection et de Géophysique, DASE, Commissariat à l'énergie atomique, Bruyères-le-Châtel, France
}

Sophie Hautot and Pascal Tarits

UMR CNRS "Domaines Océaniques", IUEM/UBO, Plouzané, France

\begin{abstract}
Electric potential variations have been recorded from November 1995 to February 1996 and continuously since October 1996 at 14 measurement points on a one $\mathrm{km}$ wide ridge separating two lakes in the French Alps. The levels of the lakes vary by several tens of meters on a yearly cycle, inducing stress variations and fluid percolation. At one point, unambiguous variations as large as $120 \mathrm{mV}$ are observed over a year, linearly correlated with the levels of the lakes with a magnitude of $2 \mathrm{mV}$ per meter of water level change. This particular measurement point lies at the edge of a SP anomaly, which supports the presence of a localized zone of ground water flow forced by the lake level, suggesting an electrokinetic mechanism. The observed correlation implies a $\zeta$-potential of the order of $-8 \mathrm{mV}$ for a 60 $\Omega m$ electrolyte, in agreement with laboratory measurements.
\end{abstract}

\section{Introduction}

Electric potential variations have been observed in some instances before earthquakes [Park et al., 1993], for example in China [Raleigh et al., 1977] or Greece [Varotsos et al., 1993]. These observations are sparse, their correlation with earthquakes in Greece is still a matter of debate [Geller et al., 1996], some may be affected by serious instrumental problems or the presence of industrial noise [Gruszow et al., 1996] and they are usually not confirmed by independent measurements using other geophysical methods. One objection to the possibility of such earthquake precursors is that no definite physical model is available to account for the observations. There is however a theoretical possibility that electrical signals can be generated in the crust through the electrokinetic effect [Mizutani et al., 1976; Bernard, 1992]. Large amounts of crustal fluids are involved during the physical processes of the earthquake cycle [e.g., Nur and Booker, 1972; Muir-Wood and King, 1993]. In addition, the electrokinetic effect has been studied in the laboratory with rock samples and variations are observed during deformation and rupture [Jouniaux and Pozzi, 1995]. Spontaneous potential (SP) anomalies are measured in geothermal fields [Corwin and Hoover, 1979] and some SP signals were shown to be related to the subsurface flows induced by the opening and closure of wells [Ishido et al., 1983]. Clear magnetic and electric signals have been observed to be associated with water flow in active volcanoes [e.g., Zlotnicki and Le Mouël, 1990; Hashimoto and Tanaka, 1995]. It appears therefore interesting to investigate further the relationship between electric potentials, stress variations and hydrogeological circulations in geophysical systems where the stress changes are

Copyright 1998 by the American Geophysical Union.

Paper number 98GL01139.

0094-8534/98/98GL-01139\$05.00 known and controlled. For example, signals have been observed on the pillar of a limestone quarry in France in relation to stress changes [Morat and Le Mouël, 1992]. Following this approach, we set up an experiment in a kilometer-size system, at a site in the French Alps located in the vicinity of artificial lakes subject to annual level variations. In this paper, the first observations are presented, and a preliminary interpretation is proposed.

\section{The site of the Sur-Frêtes experiment}

The site is located in the French Alps, $30 \mathrm{~km}$ south-west of Mont Blanc, at the geological contact between the Belledone crystalline basement and Mesozoic sedimentary layers (Figure 1). Artificial lakes are created by two dams set against the crystalline rocks: the Roselend lake $\left(187 \times 10^{6} \mathrm{~m}^{3}\right)$, and the smaller Gittaz lake $\left(13 \times 10^{6} \mathrm{~m}^{3}\right)$ which communicates with the Roselend lake through an underground pipe. The electrical measurements are done across the Sur-Frêtes ridge which separates the two lakes (Figure 1). This ridge has a complicated geological structure, and involves several highly tectonized Permo-Trias sedimentary units, lying uncorformably on the Belledone crystalline basement and overthrust by Jurassic calcareous sheets. Structures trend $\mathrm{N} 30^{\circ} \mathrm{E}$ and dip about $50^{\circ}$ to the east. Highly contrasted hydrogeological and physical properties are observed along the ridge, as confirmed by VLF and SP geophysical surveys.

The levels of both lakes vary by several tens of meters on a yearly cycle, inducing stress variations and fluid percolation. The yearly amplitude of the deformation measured with a tiltmeter installed in a tunnel located in the gneiss $1.5 \mathrm{~km}$ south-west of the Sur-Frêtes ridge amounts to $5 \mu \mathrm{rads}$. The site is covered with snow, and is not accessible from December to May. The lake level cycle is correlated with the yearly meteorological cycle only during the snow melting time at the end of spring. The lake level regression is artificially induced since it depends on the needs of electric power production.

\section{The Sur-Frêtes electric potential array}

The electrical array consists of 14 measurement points. Two measurement points were set on the floor of the lakes in order to be as close as possible to the sources of deformation. On the ridge, the measurement points are located near geological contacts in order to benefit from possible amplifications resulting from resistivity contrasts. In particular, a large static SP anomaly of $-600 \mathrm{mV}$ with respect to the reference point $\mathrm{BC}$ was found to be associated with the coal bearing sandstone, and this anomaly and its borders were instrumented. The electrical potentials are measured using second generation $\mathrm{Pb} / \mathrm{PbCl}_{2} /$ kaolinite electrodes [Petiau, 1996], installed in a 1.2 to $1.5 \mathrm{~m}$ deep $40 \mathrm{~cm}$ diameter holes in 30 liters of salted clay. This set-up was tested in Garchy (France) during an international intercomparison experiment, and 


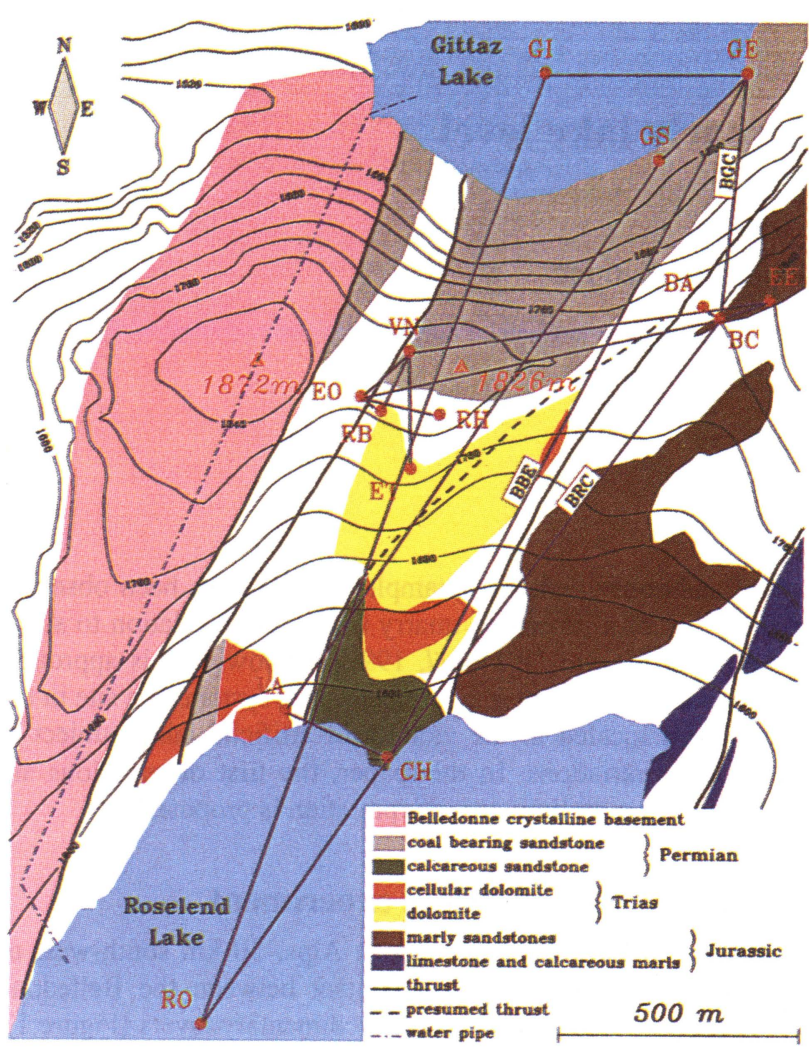

Figure 1. Geological map of the Sur-Frêtes ridge and the electric potential array. Electric potential measurement points are identified by two letters.

was demonstrated to provide a stability of the order of $1 \mathrm{mV}$ per year with a drift noise varying between 0.4 and $0.8 \mathrm{mV}$ per month [Perrier et al., 1997]. The electrodes are combined into 20 dipoles in order to provide redundancy. Several measurements are made in the same hole with independent electrodes. Electric potential resulting from the evolution of the electrode itself or of its interface with the soil can be identified when potential differences on a closed loop are compared. For example, the combination RTNS=BBE+BGC-BRC cancels all real electrical signals and contains the sum of all drift artifacts of the 6 independent electrodes of the BBE, BGC and BRC dipoles (Figure 1). All electrical signals are connected to a measuring station located on the ridge. Potential differences are read by high input-impedance amplifiers, filtered with a corner frequency of $33 \mathrm{mHz}$, and digitized at a frequency of $0.1 \mathrm{~Hz}$. One minute averages are stored and transmitted to the laboratory every day by a radio-phone link. Meteorological parameters are registered with a sampling rate of 5 minutes. In this paper, electric data are further low-pass filtered at 2 days.

\section{First observations}

The experiment, installed in October 1995, recorded its first data in November 1995. From March 1996, some cables were damaged and had to be repaired. The data have been recorded continuously since October 1996. Some dipoles show strong yearly variations. In Figure 2, three potential differences are displayed as a function of time: the $540 \mathrm{~m}$-long BGC dipole, the $1.1 \mathrm{~km}$-long $\mathrm{BRC}$ dipole and the $1.5 \mathrm{~km}$-long BBE dipole, as well as the combination RTNS. Over the October 1996 to September 1997 period, variations of the order of $120 \mathrm{mV}$ are observed on BBE and BRC dipoles, and of the order of $20 \mathrm{mV}$ on BGC. The RTNS combination is stable over a year within better than $1 \mathrm{mV}$. This indicates that the observed signals are real, and that the electric potential data, with stabilities comparable or better than measured in Garchy [Perrier et al., 1997], are of excellent quality. The other recorded dipoles show smaller time variations except the two dipoles pointing to the $\mathrm{CH}$ point (BBR and LRB, Figure 1). This implies that it is the potential of the $\mathrm{CH}$ point with respect to the other potential measurement points that exhibits the majority of the variation. This is also true for the first part of the data from November 1995 to February 1996 (Figure 2) with a similar amplitude, demonstrating that, although only one complete yearly cycle is available for the moment, the phenomenon is remarkably reproducible. The $\mathrm{CH}$ point is located on the bank of the lake, close to the maximum level of the Roselend lake $(1557 \mathrm{~m})$, on the edge of a steep reef of calcareous sandstone (Figure 1). The potential of the point $\mathrm{CH}$ is clearly related to the level of the Roselend lake, also shown in Figure 2. The correlation is nearly linear, with a variation of about $1.6 \mathrm{mV}$ per meter of water level change during the first period and 2.4 to $2.8 \mathrm{mV}$ per meter of water level change from October 1996 to September 1997. It is interesting to note that the order of magnitude of this effect is in agreement with the value of 1.4 $\mathrm{mV} / \mathrm{m}$ obtained by Gex [1980], who related the maximum SP measured below seven dams to their water level. However, there is no further electrical variations when the level of the Roselend lake is below $1507 \mathrm{~m}$ (Figure 2).
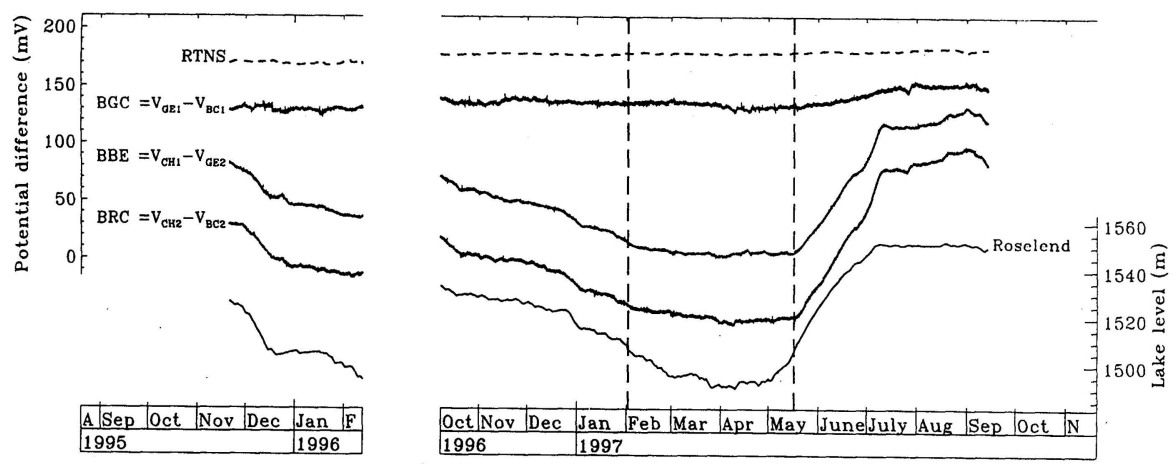

Figure 2. Potential differences for the BGC, BRC and BBE dipoles as a function of time. The variable RTNS is the combination BBE+BGC-BRC. The averages values of BGC, BRC, BBE and RTNS are arbitrary. The signals are low-frequency filtered above two days. The dashed lines represent the level below which no correlation is observed between the electric potential and the water level. 


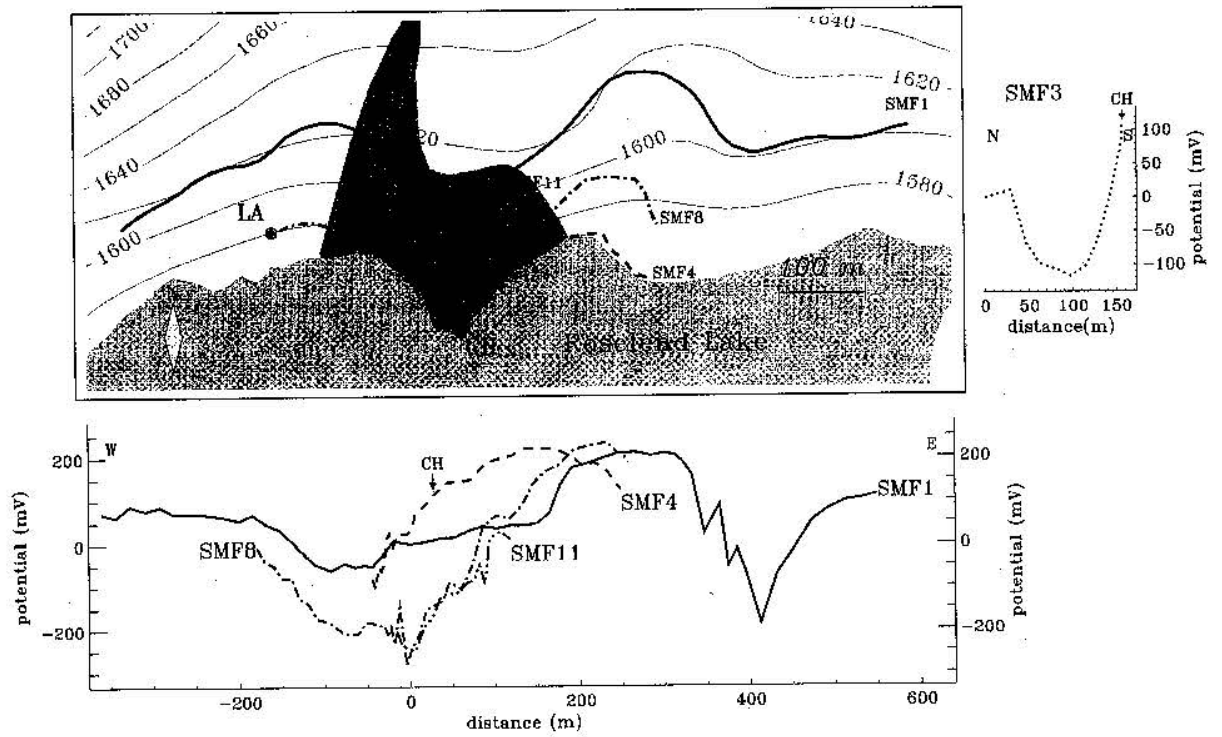

Figure 3. Self-Potential survey of July 1997. The reference point is indicated by the square. The shaded area corresponds to the calcareous sandstone formation (Figure 1).

\section{Interpretation}

The electric potential variation at point $\mathrm{CH}$ requires the variation of either an electrical source, or of the resistivity of the medium between a constant electrical source and the measurement point. The resistivity of the medium could vary through the change of the level of the water, which has a resistivity of $55 \Omega \mathrm{m}$. However, in that case, we would expect an observable signal on other points, and in particular at the RO lake point and the LA point near the Roselend lake (Figure 1). Also, there would be no reason why the variation of the electrical potential would be linearly correlated with the water level. Since the electrical signal is large near the $\mathrm{CH}$ point only, we will rather assume that an electrical source exists in the neighborhood of this point, and that its intensity must be proportional to the water level of the lake by some mechanism.

A detailed SP survey was performed in July 1997 around the $\mathrm{CH}$ point, when the Roselend lake was at its highest level (Figure 3). Spatial SP variations roughly follow the geological structures, but one can see an additional strong anomaly within the Permian calcareous sandstone formation (see also Figure 1), with a radius of about 100 meters and a magnitude of $-200 \mathrm{mV}$ (profile SMF3). The point $\mathrm{CH}$ is on the edge of this anomaly, so that it probably monitors its time variations. Our measurements suggest that this anomaly is small when the level of lake is high, and large when the level of the lakes is low. We therefore infer the presence of an

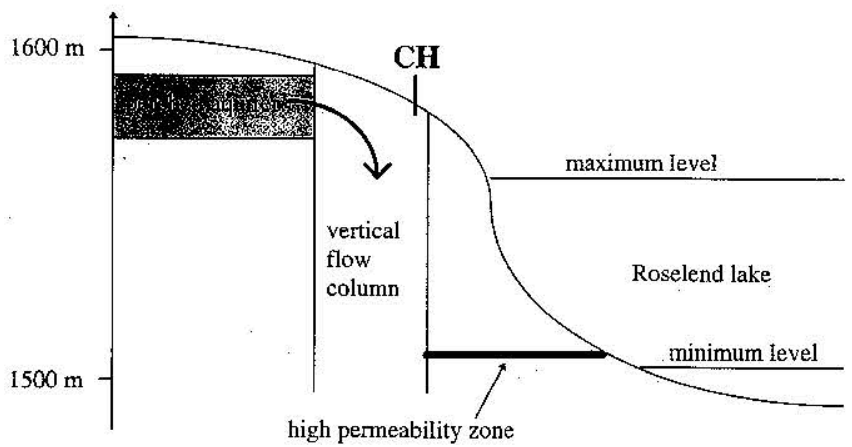

Figure 4. Preliminary interpretation for the time variation of the electric potential at point $\mathrm{CH}$. electrokinetic source associated with a ground water flow modulated by the lake water level.

Hydrogeological investigations performed before the construction of the Roselend dam support the presence of ground water flow $[E D F, 1951]$. Perched aquifers were found in a borehole located in the nearby cellular dolomite formation. A spring located at an altitude of $1480 \mathrm{~m}$, that was probably related to the aquifers sampled by the borehole, indicated a large water discharge associated with a stable water temperature. Level variations in the borehole were reported, indicating a relatively high permeability of the formations.

These observations lead to the following conceptual model, sketched in Figure 4. The SP anomaly near point $\mathrm{CH}$ in Figure 3 corresponds to an underground vertical column of percolating fluid, creating streaming potentials. The vertical column has a permeability such that a constant level of the interstitial water is maintained from a higher aquifer. The bottom part of the column is connected to the lake through a high permeability zone, which constrains the pressure at the bottom of the column to be equal to the water level in the lake. When the lake is at the level corresponding to the piezometric level in the column, there is no pressure gradient in the column and no potential.

When the lake level is lower than the stable piezometric level in the column by a height $\Delta h$, there is a streaming potential producing a negative potential above the column.

For the purpose of getting a first order estimate of the maximum effect, the leakage current through the medium in which the vertical column is inserted will be neglected. The magnitude of the potential drop along the column is then given by $C_{s} \rho g \Delta h$, where $C_{s}$ is the streaming potential coefficient of the rock, and $\rho$ is the water density. As the upstream of flow in rocks containing quartz is usually negatively charged $\left(C_{s,}\right.$ positive, e.g., [Morgan et al., 1989]), the generated potential has the proper sign to explain the observations. In addition, when the water is below the exit point of the high permeability zone (Figure 4), there is no more effect on the potential. The exit point of the high permeability zone in Figure 4 could actually be associated with a source at an altitude of $1503 \mathrm{~m}$, indicated on early maps. Such a simple model accounts qualitatively for the features of the data.

The value of the streaming potential coefficient can be estimated from the observed correlation. If one can assume that 
the potential of the point $\mathrm{CH}$ is representative of the potential at the top of the column with respect to infinity, then the potential drop along the vertical column is of the order of $40 \mathrm{mV} / 0.1 \mathrm{MPa}$. The temperature of the water column is about $7^{\circ} \mathrm{C}$, typical value of the spring waters of the site. Assuming the temperature dependence given by Morgan et al. [1989], the streaming potential coefficient at $25^{\circ} \mathrm{C}$ would then be $34 \mathrm{mV} / 0.1 \mathrm{MPa}$. From this value, it is useful to extract the value of the $\zeta$-potential which characterizes the mineral surface properties. It is related to the streaming potential by $C_{s}=-\varepsilon \zeta \rho / \eta$. where $\varepsilon$ is the water permittivity, $\eta$ its dynamic viscosity and $\rho_{f}$ its resistivity [e.g., Mizutani et al., 1976; Morgan et al., 1989]. We obtain a $\zeta$ potential of $-8 \mathrm{mV}$ for $\rho_{f}=60 \Omega \mathrm{m}$ at $25^{\circ} \mathrm{C}$.

The $\zeta$ potential of various crushed samples from the Sur-Frêtes site has been measured in the laboratory [Lorne et al., submitted to J. Geophys. Res., 1998]. For the Permian calcareous sandstone constituting the reef at point $\mathrm{CH}$, a value of $-8.6 \mathrm{mV}$ has been measured at $25^{\circ} \mathrm{C}$ for a $\mathrm{KCl}$ solution with $\rho_{f}=300 \Omega \mathrm{m}$ and $p H=5.7$. This value can be translated to the field conditions using scaling relations obtained experimentally. For $\rho_{f}=60 \Omega \mathrm{m}, p H=7$ and a typical mineral water ion composition, the $\zeta$-potential becomes $-4.7 \mathrm{mV}$. The agreement between this value, and the value of $-8 \mathrm{mV}$ inferred from the field, is strongly supporting the proposed interpretation.

\section{Implications for earthquake precursors}

More work is needed to assess whether the observations presented in this paper are relevant to the geophysical processes induced by the preparation of an earthquake. It is however interesting to note that the signals observed on the Sur-Frêtes site are local : they are mainly associated with a single heterogeneous formation. Large effects are not observed on other points of the electrical array. The yearly stress variations of the Sur-Frêtes ridge can be estimated assuming an elastic half-space, and are of the order of $10^{4} \mathrm{~Pa}$. This is similar for example to the yearly increase of stress in the neighborhood of a fault where an earthquake of magnitude 7 occurs every about hundred years [Scholz, 1990]. So this experiment may be useful to assess the electrokinetic effect that could be expected in the vicinity of a fault during stress accumulation. If an electrical effect is to be produced at large distance from the earthquake source, one has to imagine a mechanism where the stress changes are transferred to large distance by a network of fractures, or a mechanism based on fluid instabilities [Bernard, 1992]. The model shown in Figure 4 is characterized by the coupling between a vertical flow column, and a high-permeability zone linking it to the reservoir. Such a structure of faults could exist in the crust, as suggested by the amounts of water released over large areas after earthquakes [Muir-Wood and King, 1993]. However, no transient effect, possibly triggered by deformation, has been observed up to now at point $\mathrm{CH}$.

Acknowledgments. This experiment was funded by the Environment Program of the Commissariat à l'Energie Atomique, and by the Ministry of Environment. The authors pay homage to G. Sellier who installed the CH measurement point, his last field work before his untimely death. G. Petiau is thanked for sharing with us his tremendous expertise on electrodes, and EDF for its water level data and access to the lakes.

\section{References}

Bernard, P., Plausibility of long distance electrotelluric precursors to earthquakes, J. Geophys. Res., 97, 17531-17546, 1992.

Corwin, R.F., and D.B. Hoover, The self-potential method in geothermal exploration, Geophysics, 44, 226-245, 1979.

Electricité de France, Service Géologie, Note géologique sur l'étanchéité de la cuvette de Roselend en direction du col de Sur-Frêtes, Report 12-1951, 1951

Geller, R., Editor, Debate on «VAN», Special issue, Geophys. Res. Lett., 23, 1291-1452, 1996.

Gex, P., Phénomènes d'électrofiltration liés à quelques sites de barrages, Bull. Soc. Vaud. Sc. Nat., 75, 39-50, 1980.

Gruszow, S., J.C. Rossignol, A. Tzanis, and J.L. Le Mouël, Identification and analysis of electromagnetic signals in Greece: the case of the Kozani earthquake VAN prediction, Geophys. Res. Let, 23, 2025$2028,1996$.

Hashimoto, T. and Y. Tanaka, A Large Self-Potential Anomaly on Unzen Volcano, Shimabara Peninsula, Kyushu Island, Japan, Geophys. Res. Let., 22, 191-194, 1995.

Ishido, T., H. Mizutani, and K. Baba, Streaming potential observations, using geothermal wells and in situ electrokinetic coupling coefficients under high temperature, Tectonophysics, 91, 89-104, 1983.

Jouniaux, L., and J.P. Pozzi, Streaming potential and permeability of saturated sandstones under triaxial stress: Consequences for electrotelluric anomalies prior to earthquakes, J. Geophys. Res., 100, 10,197-10,209, 1995.

Morat, P., and J.L. Le Mouël, Electrical signals generated by stress variations in porous non saturated rocks, C. R. Acad: Sci. Paris, 315, 955-963, 1992

Morgan, F.D., E.R. Williams, and T.R. Madden, Streaming potential properties of westerly granite with applications, J. Geophys. Res., 94, 12,449-12,461, 1989.

Muir-Wood, R., and G.C.P. King, Hydrological Signatures of Earthquake Strain, J. Geophys. Res., 98, 22,035-22,068, 1993.

Mizutani, H., T. Ishido, T. Yokokura, and S. Ohnishi, Electrokinetic phenomena associated with earthquakes, Geophys. Res. Lett., 3, 365$368,1976$.

Nur, A., and J.R. Booker, Aftershocks caused by fluid flow, Science, 175, $885-887,1972$

Park, S.K., M.J.S. Johnston, T.R. Madden, and H.F. Morrison, Electromagnetic Precursors to Earthquakes in the ULF band: a review of Observations and Mechanisms, Rev. Geophys., 31, 117-132, 1993.

Perrier, F. , G. Petiau, G. Clerc, V. Bogorodsky, E. Erkul, L. Jouniaux, D. Lesmes, J. Macnae, J. Meunier, D. Morgan, D. Nascimento, G. Oettinger, G. Schwarz, H. Toh, M. Valiant, K. Vozoff, and O YaziciÇakiin, A one-year systematic study of electrodes for long period measurement of the electric field in geophysical environments, $J$. Geomag. Geoelec., 49, 1677-1696, 1997.

Petiau, G., $\mathrm{Pb} / \mathrm{PbCl} 2$ Electrodes: second generation. Proiceedings of the Workshop "Electrodes", Garchy, April 24-29, 1995 (Edited by G. Clerc, F. Perrier, G. Petiau, and M. Menvielle), 49-51, 1996.

Raleigh, B., P. Molnar, T. Hanks, A. Nur, J. Savage, H. Craig, R. Turner, and $G$. Bennett, The prediction of the Haicheng earthquake, EOS Trans. Am. Geophys. Union, 58, 236-272, 1977.

Scholz, C.H., The Mechanics of Earthquakes and Faulting, 439 pp., Cambridge University Press, 1990.

Varotsos, P., K. Alexopoulos, and M. Lazaridou, Latest aspects of earthquake prediction in Greece by seismic electric signals, II, Tectonophysics, 224, 1-37, 1993.

Zlotnicki, J., and J.L. Le Mouël, Possible electrokinetic origin of large magnetic variations at La Foumaise Volcano, Nature, 343, 633-636, 1990.

(Received November 6, 1997; revised February 13, 1998; accepted March 20, 1998) 